\title{
Research Article \\ COPB2: A Novel Prognostic Biomarker That Affects Progression of HCC
}

\author{
Jiayao Zhang $\mathbb{D}^{1},{ }^{1}$ Xiaoyu Wang, ${ }^{1}$ Guangbing Li, ${ }^{1,2}$ Jingyi He, ${ }^{1,2}$ Ziwen Lu, $^{1}$ Yang Yang, \\ Yong Jiang, ${ }^{1}$ Liyong Jiang, ${ }^{1}$ Feiyu Li, ${ }^{1}$ and Jun Liu ${ }^{10,2}$ \\ ${ }^{1}$ Department of Hepatobiliary Surgery and Center of Organ Transplantation, Shandong Provincial Hospital, Cheeloo College \\ of Medicine, Shandong University, Jinan, Shandong 250021, China \\ ${ }^{2}$ Department of Hepatobiliary Surgery and Center of Organ Transplantation, Shandong Provincial Hospital Affiliated to Shandong \\ First Medical University, Jinan, Shandong 250021, China
}

Correspondence should be addressed to Jun Liu; dr_liujun1967@126.com

Received 14 December 2020; Revised 4 February 2021; Accepted 6 March 2021; Published 22 March 2021

Academic Editor: Junyan Tao

Copyright (C) 2021 Jiayao Zhang et al. This is an open access article distributed under the Creative Commons Attribution License, which permits unrestricted use, distribution, and reproduction in any medium, provided the original work is properly cited.

Purpose. This study is aimed at investigating the expression, underlying biological function, and clinical significance of coatomer protein complex subunit beta 2 (COPB2) in hepatocellular carcinoma (HCC). Methods. HCC-related data were extracted from The Cancer Genome Atlas (TCGA) database, International Cancer Genome Consortium (ICGC) database, and Gene Expression Omnibus (GEO) database. A logistic regression module was applied to analyze the relationship between the expression of COPB2 and clinicopathologic characteristics. The Cox proportional hazard regression model and Kaplan-Meier method were used for survival analysis. Gene set enrichment analysis (GSEA) was used to annotate the underlying biological functions. Loss-of-function experiments were conducted to determine the underlying mechanisms. Results. COPB2 was overexpressed in HCC, and high expression of COPB2 was significantly correlated with higher alpha fetoprotein (AFP) (odds ratio (OR) $=1.616,>20$ vs. $\leq 20$, $p<0.05)$, stage ( $\mathrm{OR}=1.744$, III vs. I, $p<0.05)$, and grade ( $\mathrm{OR}=1.746, \mathrm{G} 4+\mathrm{G} 3$ vs. $\mathrm{G} 2+\mathrm{G} 1, p<0.05)$. Kaplan-Meier survival analysis showed that HCC patients with high COPB2 expression had a worse prognosis than those with low COPB2 expression ( $p<0.0001$ for TCGA cohort, $p<0.05$ for ICGC cohort). The univariate Cox (hazard ratio $(\mathrm{HR})=1.068, p<0.0001)$ and multivariate Cox $(\mathrm{HR}=2.011, p<0.05)$ regression analyses suggested that COPB2 was an independent risk factor. GSEA showed that $\mathrm{mTOR}$ and other tumor-related signaling pathways were differentially enriched in the high COPB2 expression phenotype. Silencing of COPB2 inhibited the proliferation, migration, and invasion abilities by suppressing epithelial-mesenchymal transition and mTOR signaling. Conclusion. COPB2 is a novel prognostic biomarker and a promising therapeutic target for HCC.

\section{Introduction}

Liver cancer is one of the most common fatal cancers, ranking sixth among cancer diagnoses, and is the fourth leading cause of cancer-related deaths, with 841,000 new cases and 782,000 deaths annually worldwide [1]. The morbidity and mortality of liver cancer rank fourth (10.6\%) and third (12.9\%), respectively, among all malignant tumors in China [2]. Hepatocellular carcinoma (HCC) accounts for approximately $80 \%$ of primary liver cancers; due to its asymptomatic disease progression and limited treatment options, it has become a leading cause of cancer burden globally $[1,3,4]$.
There are several ways to treat HCC, such as surgical resection, locoregional therapy, liver transplantation, and systemic therapy; however, its prognosis remains poor, and its survival rate is much lower for patients with metastasis and recurrence [5-8]. Therefore, finding new biomarkers is very important for the prognosis and treatment of HCC and will benefit more patients.

The coatomer protein complex subunit beta 2 (COPB2), encoded by a gene located on chromosome 3q23 [9], is one of the seven subunits that form coatomer complex I (COPI), which is one of the three types of coat proteins (COPs) that play a key role in intracellular transport by forming transport 
vesicles [10]. Previous studies have reported that the main functions of COPB2 are the regulation of extracellular membrane transport and mediation of retrograde transport from the Golgi complex to the endoplasmic reticulum (ER) [1113]. Recently, COPB2 was reported to have important correlations with various cancer types and has different functions in different tumors, such as breast cancer, glioma, and prostate cancer [14-16]. Silencing COPB2 can inhibit the proliferation of colon cancer cells by inducing cell cycle arrest [17]. In addition, coatomer protein complex subunit alpha (COPA), another subunit of COPI, is an important paralog of COPB2 [18] and has been reported to be upregulated in tumors relative to paired adjacent nonmalignant tissues in patients with liver cancer [19]. It was also reported that reduced editing of COPA was implicated in the pathogenesis of HCC and editing of COPA ${ }^{W T}$ may switch it from a tumorpromoting gene to a tumor suppressor by deactivating the $\mathrm{PI} 3 \mathrm{~K} / \mathrm{AKT} / \mathrm{mTOR}$ pathway through downregulation of caveolin-1 (CAV1) [20]. Deregulated mTOR signaling significantly contributes to the molecular pathogenesis of HCC [21]. Considering the relationship between COPB2 and other tumors and the role of its paralog, COPA, in HCC, we hypothesized that COPB2 may play an important role in the progression of HCC and may be a new potential therapeutic target for HCC. By querying the online UALCAN database (http:// ualcan.path.uab.edu/analysis.html), we found that COPB2 expression was elevated in HCC and correlated with its prognosis [22], but no research has yet revealed the mechanism by which COPB2 regulates the malignant progression of HCC.

In this study, we explored the role of COPB2 in HCC by analyzing HCC-related data from The Cancer Genome Atlas (TCGA) database, the International Cancer Genome Consortium (ICGC) database, and Gene Expression Omnibus (GEO) databases, as well as conducting a series of experiments. The results of the current study revealed that COPB2 is a novel prognostic biomarker and a promising therapeutic target for HCC.

\section{Material and Methods}

2.1. Bioinformatics Analysis. All HCC-related data (including clinical information and corresponding mRNA expression data) were downloaded from The Cancer Genome Atlas (TCGA) database (https://portal.gdc.cancer.gov/repository) and the International Cancer Genome Consortium (ICGC) database (https://dcc.icgc.org/releases). We then used Perl ( $v$ 5.26.3) and $\mathrm{R}(\mathrm{v}$ 3.6.3) to sort and extract the data and merged the expression data with clinical data. Cases without clinical data or expression data were excluded. At the same time, patients with a pathological type other than primary hepatocellular carcinoma were also excluded; 370 HCC cases from TCGA cohort and 232 HCC cases from the ICGC cohort were used for subsequent analysis. The patients' basic information is shown in Tables S1 and S2. The clinical characteristics of TCGA cohort included age, sex, body mass index (BMI), $\mathrm{T}$ stage, lymph node $(\mathrm{N})$, metastasis (M), stage, grade, tumor status, family history of cancer, vascular invasion, AFP, new tumor event, survival status, and survival time, while the ICGC cohort included age, sex, stage, grade, and tumor status. Cases with incomplete clinical pathological information were included in the analysis based on the available clinical information and excluded from the analysis of the clinical pathological features where data were missing. In the survival analysis, patients with a survival time of less than 30 days were excluded, since they may have died of serious complications (including bleeding, intracranial infections, and heart failure) rather than HCC. To further verify the expression level of COPB2 mRNA in patients with HCC, six datasets from the Gene Expression Omnibus (GEO) database were used (Table S3). In the present study, in addition to difference analysis and Kaplan-Meier analysis, the logistic regression and Cox proportional hazard regression models were used for clinical correlation analysis and survival analysis, respectively. Gene set enrichment analysis (GSEA) is a method to identify classes of genes or proteins that are overrepresented in a large set of genes or proteins and may be associated with disease phenotypes [23]. GSEA was used to explore the potential biological signaling pathways related to COPB2 in HCC. During each analysis, all genes were generated in an ordered list and were classified into high and low COPB2 expression phenotypes. Gene set permutations were performed 1000 times. A nominal $p$ value $<0.05$ and false discovery rate $(\mathrm{FDR})<0.05$ were used to filter the pathways enriched in each phenotype.

2.2. Cell Culture and siRNA Transfection. BEL7402 and SMMC7721 HCC cell lines were purchased from the BeNa Culture Collection (Beijing, China). All cells were cultured in RPMI 1640 medium supplemented with 10\% fetal bovine serum (FBS) in a humidified chamber with $5 \% \quad \mathrm{CO}_{2}$ at $37^{\circ} \mathrm{C}$. siRNA for COPB2 was purchased from Genomeditech (Shanghai, China). BEL7402 and SMMC7721 cells were seeded in six-well plates at 30-50\% confluence and were then transfected with $50 \mathrm{nmol} / \mathrm{L}$ siRNAs using Lipofectamine 3000 reagent (Thermo, L3000015, Waltham, MA, USA). The cells were collected following transfection efficiency determination and follow-up experiments after being transfected for 48-72 hours.

2.3. CCK-8 Assay. The transfected BEL7402 and SMMC7721 cells were seeded and cultured in four 96-well plates at 2000 cells/well, with five replicate wells for each group; the cell viability of each group was measured after $0 \mathrm{~h}, 24 \mathrm{~h}, 48 \mathrm{~h}$, and $72 \mathrm{~h}$ at a wavelength of $450 \mathrm{~nm}$ with a microplate reader after adding CCK-8 reagent for $3 \mathrm{~h}$ in each well.

2.4. Immunohistochemistry. Tumor tissues and corresponding adjacent nontumor tissues in 20 HCC patients undergoing hepatectomy were fixed with $4 \%$ paraformaldehyde immediately after isolation and then embedded in paraffin for being cut into $5 \mu \mathrm{m}$ thick continuous sections. These sections were then deparaffinized, hydrated, and incubated overnight with the primary rabbit anti-COPB2 polyclonal antibody (Abcam, ab192924, CA, USA) and primary rabbit anti-phospho-mTOR (Ser2448) polyclonal antibody (CST, 2796, MA, USA) overnight at $4^{\circ} \mathrm{C}$. On the next day, the cells were incubated with the secondary antibody at $37^{\circ} \mathrm{C}$ and then 
visualized using a DAB kit (ZSGB-BIO, ZLI-9017, Beijing, China) and counterstained with hematoxylin. The expression level was independently evaluated by two senior pathologists using the $\mathrm{H}$-score method.

2.5. Scratch Wound Healing Assay. The transfected BEL7402 and SMMC7721 cells were seeded in six-well plates. When they reached approximately $80-90 \%$ confluence, the cells were scratched using a $200 \mu \mathrm{L}$ pipette tip. Serum-free medium was added after washing with phosphate-buffered saline to remove debris. Photographs were taken at $0 \mathrm{~h}$ and $48 \mathrm{~h}$ to compare wound healing rates.

2.6. Transwell Assay. For the migration assay, $4 \times 10^{4}$ cells ( $200 \mu \mathrm{L}$ serum-free cell suspension) were seeded into the upper Transwell chamber with $8 \mu \mathrm{m}$ pore inserts (Corning, NY, USA), while the bottom chamber was filled with $600 \mu \mathrm{L}$ RPMI 1640 medium supplemented with 10\% fetal bovine serum. After incubation at $37^{\circ} \mathrm{C}$ with $5 \% \mathrm{CO}_{2}$ for $24 \mathrm{~h}$, cells invading the lower surfaces were fixed with $4 \%$ paraformaldehyde and stained with $0.1 \%$ crystal violet stain solution, while cells on the upper surface were scraped. Nine random fields were used for statistical analysis. For the invasion assay, $1 \times 10^{5}$ cells $(200 \mu \mathrm{L}$ serum-free cell suspension) were seeded into the upper Transwell chamber, which was prepaved with Matrigel. The remaining steps were the same as those for the migration assay.

2.7. Cell Cycle Distribution. All cells were collected, fixed, and stained after being transfected for $72 \mathrm{~h}$, and the cell cycle distribution was detected using a Muse Cell Analyzer (Merck \& Millipore, Germany). All experimental procedures were performed in accordance with the manufacturer's protocol.

2.8. Western Blotting. The total protein in each group of cells was lysed in PIPA lysis buffer (Solarbio, R0010) supplemented with phenylmethylsulfonyl fluoride (PMSF) protease inhibitor (Thermo Scientific, 36987, Waltham, MA, USA) and phosphatase inhibitor (Thermo Scientific, 78428, Waltham, MA, USA); and their concentrations were then measured using a BCA Protein Assay Kit (Solarbio, PC0020, Beijing, China). $30 \mu \mathrm{g} /$ well of protein extracts was separated on $10 \%$ SDS-PAGE and transferred onto Nitrocellulose Transfer Membrane (PALL, 66485, NY, USA). After cutting into different strips according to the molecular weight of target proteins, the membranes were reacted with primary antibodies against target proteins overnight on a shaker at $4^{\circ} \mathrm{C}$. On the next day, these bands were visualized after incubation with the secondary antibody.

2.9. Statistical Analysis. GraphPad Prism 8.0, SPSS 25.0, and $\mathrm{R}$ 3.6.3 software were used for all statistical analyses. The distribution of all data was tested for normality prior to statistical analysis. When comparing the differences between two groups, we used the $t$-test for normally distributed data and used a nonparametric test (unpaired: Mann-Whitney $U$ test; paired: Wilcoxon matched-pairs signed rank-test) for the data that were not normally distributed. The data of CCK-8 assays was analyzed using two-way repeated measurement ANOVA with Sidak's multiple comparisons test. Survival was analyzed using a Kaplan-Meier plot and log-rank test. The correlation analysis between COPB2 expression level and clinicopathological parameters in HCC patients used logistic regression. The correlation between different clinicopathological variables and overall survival was explored using univariate and multivariate Cox proportional hazard regression mode. $p<0.05$ was considered to be a significant statistical difference.

\section{Results}

3.1. COPB2 Overexpressed in HCC. The mRNA expression data of $370 \mathrm{HCC}$ tissues and 50 matched nontumor tissues from TCGA cohort were analyzed. The results showed that COPB2 mRNA was significantly overexpressed in HCC tissues compared with the expression in nontumor tissues using unpaired and paired tests (Figure 1(a); unpaired: $p<0.0001$, paired: $p<0.0001)$. For the ICGC cohort, 232 HCC tissues and 199 matched nontumor tissues were analyzed, and the results were consistent with those of TCGA cohort (Figure 1(b); unpaired: $p<0.0001$, paired: $p<0.0001)$. To further verify the expression level of COPB2 mRNA in patients with HCC, six datasets from the GEO database were analyzed and similar results were obtained: GES76427 (tumor $=115$, nontumor $=$ 52) (Figure 1(c); unpaired: $p<0.0001$, paired: $p<0.0001$ ), GSE14520 (tumor $=225$, nontumor $=220) \quad($ Figure $1(\mathrm{~d})$; unpaired: $p<0.0001$, paired: $p<0.0001$ ), GSE39791 (tumor $=72$, nontumor $=72$ ) (Figure S1A; unpaired: $p<0.0001$, paired: $p<0.0001)$, GES36411 (tumor $=42$, nontumor $=42$ ) (Figure S1B; unpaired: $p<0.001$ ), GSE102079 (tumor $=152$, nontumor $=105) \quad($ Figure S1C; unpaired: $p<0.01)$, and GSE25097 (tumor $=268$, nontumor $=289$ ) (Figure S1D; unpaired: $p<0.001)$. In order to verify the results of the above bioinformatics analysis, we performed immunohistochemical staining on tumor tissues $(n=20)$ and matched nontumor tissues $(n=20)$ from HCC patients; as expected, the results showed that COPB2 was significantly overexpressed in tumor tissues (Figures 1(e) and 1(f), $p<0.0001$ ).

\subsection{High COPB2 Expression Was Correlated with Poor} Prognosis in HCC Patients. We conducted a further correlation analysis on the expression data and clinical data of HCC cases from TCGA and ICGC databases. The results indicated that high expression levels of COPB2 positively correlated with the clinical characteristics of poor prognosis. There were significant differences in COPB2 expression between different subgroups defined based on AFP $(\leq 20$ $(n=147)$ vs. $>20(n=130), p<0.05), \mathrm{T}$ stage (T2 $(n=93)$ vs. T1 $(n=181), p<0.05$; T3 $(n=80)$ vs. T1 $(n=181), p<$ $0.05)$, stage (Stage III $(n=85)$ vs. Stage I $(n=171), p<0.01)$, and grade $(\mathrm{G} 3(n=121)$ vs. G1 $(n=55), p<0.05 ; \mathrm{G} 3$ $(n=121)$ vs. G2 $(n=177), p<0.01)$ in TCGA cohort (Figures $2(\mathrm{a})-2(\mathrm{~d}))$ and stage (Stage IV $(n=19)$ vs. Stage I $(n=36), p<0.01$; Stage IV $(n=19)$ vs. Stage II $(n=106)$, $p<0.05$; Stage III $(n=71)$ vs. Stage I $(n=36), p<0.05)$ and grade $(\mathrm{G} 3(n=58)$ vs. G1 $(n=32), p<0.001 ; \mathrm{G} 2$ $(n=121)$ vs. G1 $(n=32), p<0.05)$ in the ICGC cohort (Figures 2(e) and 2(f)). Meanwhile, a logistic regression 


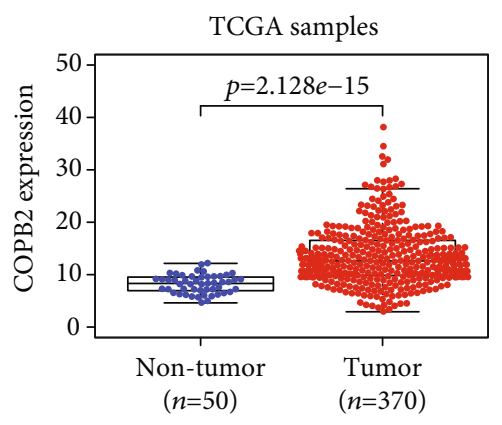

(a)

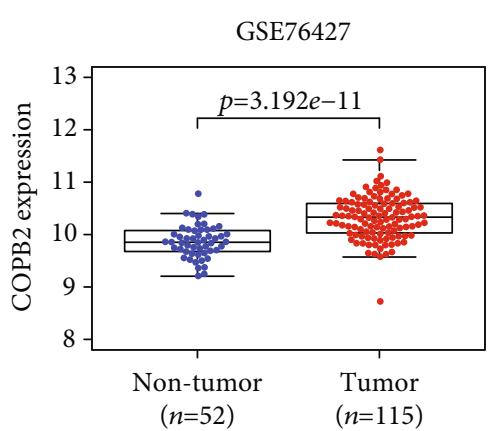

(c)
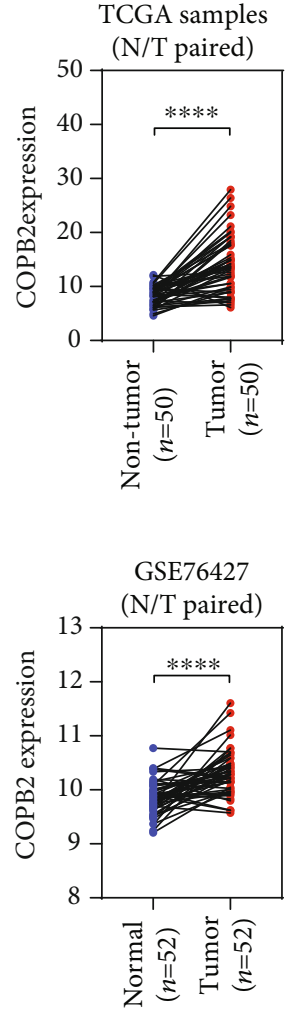

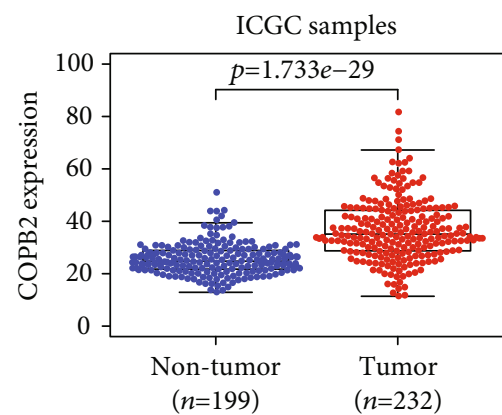

(b)

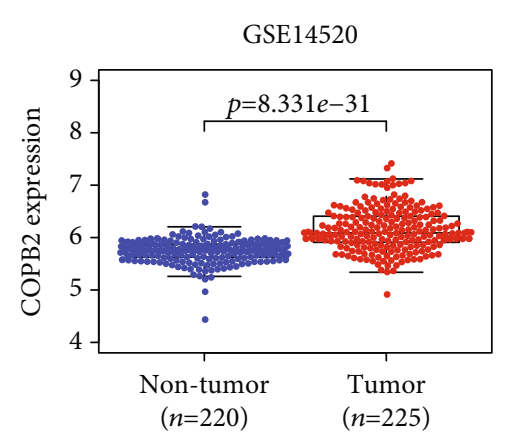

ICGC samples

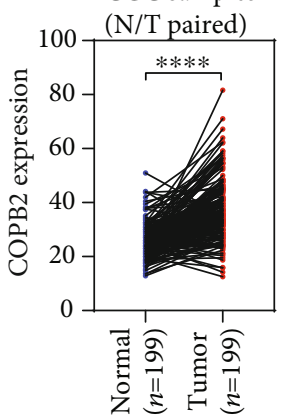

)

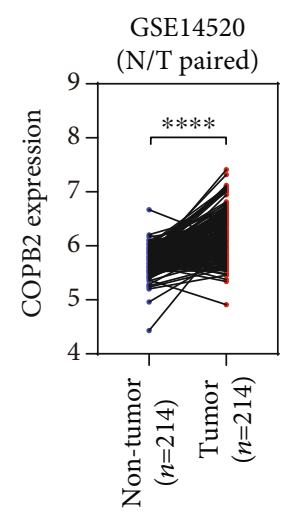

(d)

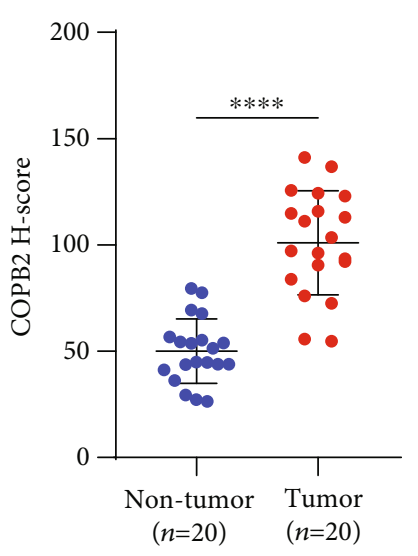

(f)

FIGURE 1: COPB2 overexpressed in HCC. (a) Comparison of COPB2 mRNA expression in tumor $(n=370)$ and nontumor tissues $(n=50)$ in patients with HCC from TCGA database using paired (Wilcoxon matched-pairs signed rank test, $p<0.0001$ ) and unpaired (Mann-Whitney $U$ test, $p<0.0001)$ analyses. (b) Comparison of COPB2 mRNA expression in tumor $(n=232)$ and nontumor $(n=199)$ tissues in patients with HCC from ICGC database using paired (Wilcoxon matched-pairs signed rank test, $p<0.0001$ ) and unpaired (Mann-Whitney $U$ test, $p<0.0001)$ analyses. (c) Comparison of COPB2 mRNA expression in tumor $(n=115)$ and nontumor tissues $(n=52)$ in patients with HCC from the GSE76472 dataset using paired (Wilcoxon matched-pairs signed rank test, $p<0.0001$ ) and unpaired (Mann-Whitney $U$ test, $p<0.0001)$ analyses. (d) Comparison of COPB2 mRNA expression in tumor $(n=225)$ and nontumor tissues $(n=220)$ in patients with HCC from the GSE76472 dataset using paired (Wilcoxon matched-pairs signed rank test, $p<0.0001$ ) and unpaired (Mann-Whitney $U$ test, $p<0.0001)$ analyses. (e, f) Immunohistochemical analysis of COPB2 in HCC tissues $(n=20)$ and adjacent nontumor tissues $(n=20)(t$-test, $p<0.0001) .{ }^{*} p<0.05,{ }^{* *} p<0.01,{ }^{* * *} p<0.001$, and ${ }^{* * * *} p<0.0001$. 


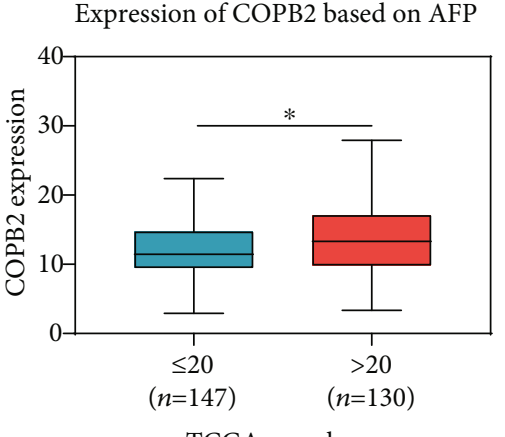

TCGA samples

(a)

Expression of COPB2 based on grade

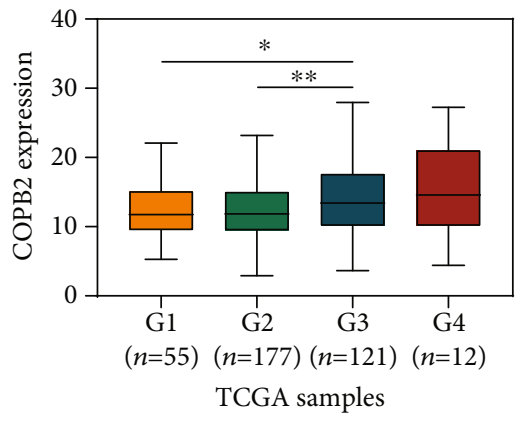

(d)

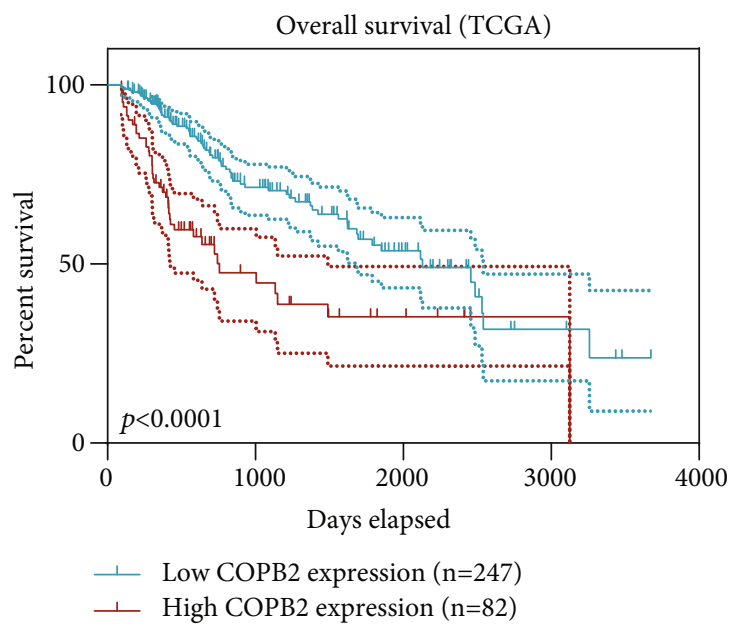

(g)

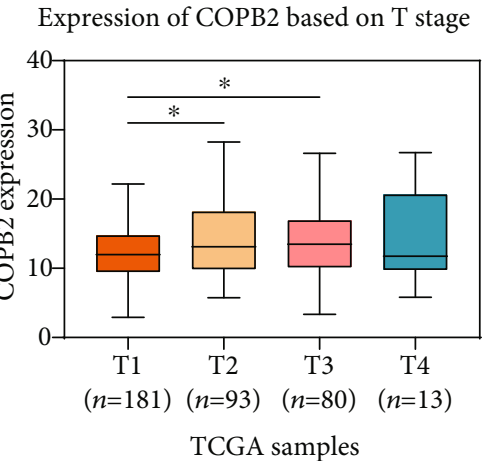

(b)

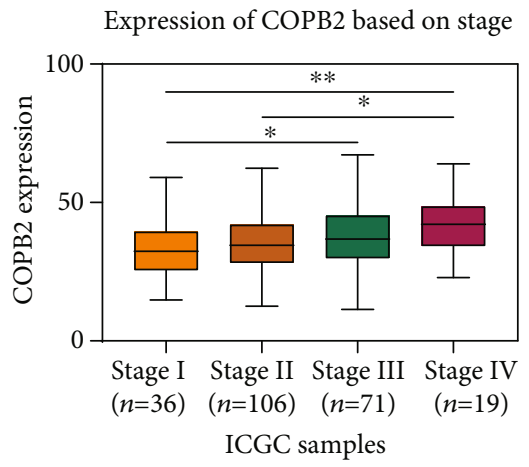

(e)

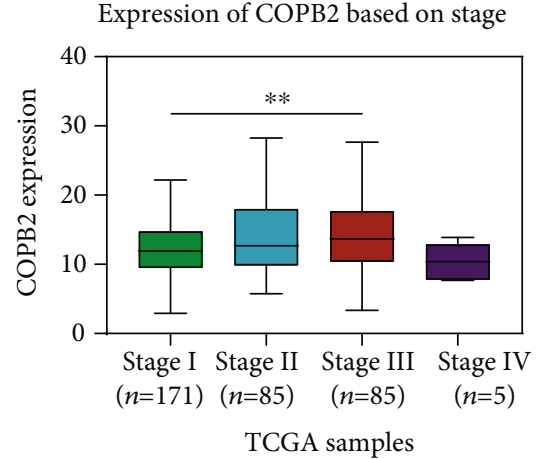

(c)

Expression of $\mathrm{COPB} 2$ based on grade

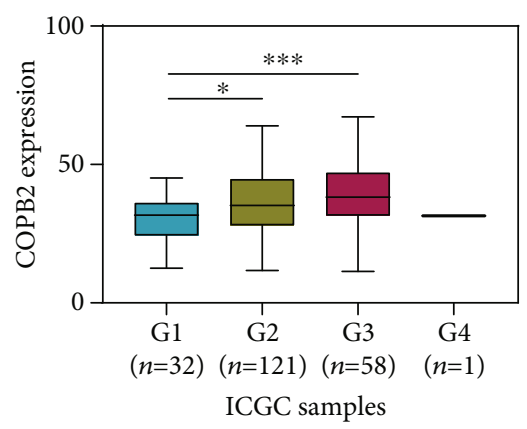

(f)

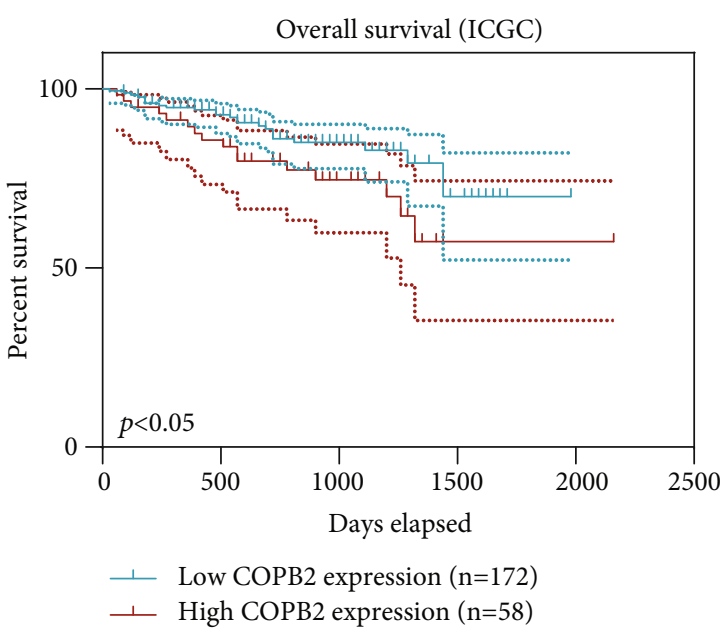

(h)

FIGURE 2: High COPB2 expression was correlated with poor prognosis in HCC patients. (a) Expression of COPB2 based on AFP in patients with HCC from TCGA cohort: AFP $\leq 20(n=147)$ vs. AFP $>20(n=130), p<0.05$ (Mann-Whitney $U$ test). (b) Expression of COPB2 based on T stage in patients with HCC from TCGA cohort: T3 $(n=80)$ vs. T1 $(n=181), p<0.05$; T2 $(n=93)$ vs. T1 $(n=181), p<0.05($ MannWhitney $U$ test). (c) Expression of COPB2 based on stage in patients with HCC from TCGA cohort: Stage III $(n=85)$ vs. Stage I $(n=171)$, $p<0.01$ (Mann-Whitney $U$ test). (d) Expression of COPB2 based on grade in patients with HCC from TCGA cohort: G3 ( $n=121)$ vs. G1 $(n=55), p<0.05$; G3 $(n=121)$ vs. G2 $(n=177), p<0.01$ (Mann-Whitney $U$ test). (e) Expression of COPB2 based on stage in patients with HCC from the ICGC cohort: Stage IV $(n=19)$ vs. Stage I $(n=36), p<0.01$; Stage IV $(n=19)$ vs. Stage II $(n=106), p<0.05$; Stage III $(n=71)$ vs. Stage I $(n=36), p<0.05$ (Mann-Whitney $U$ test). (f) Expression of COPB2 based on grade in patients with HCC from the ICGC cohort: G3 $(n=58)$ vs. G1 $(n=32), p<0.001$; G2 $(n=121)$ vs. G1 $(n=32), p<0.05$ (Mann-Whitney $U$ test). (g, h) Effect of COPB2 expression level on overall survival of HCC patients in TCGA $(p<0.0001)$ and ICGC $(p<0.05)$ cohorts (cutoff: upper quartile) (Kaplan-Meier plot and logrank test). ${ }^{*} p<0.05,{ }^{* *} p<0.01,{ }^{* * *} p<0.001$, and ${ }^{* * * *} p<0.0001$.

analysis of TCGA cohort also revealed similar results (Table 1).

Kaplan-Meier survival analysis (cases with a survival time of less than 30 days were not considered) indicated that
HCC patients with high COPB2 expression had a more unfavorable prognosis than those with low COPB2 expression in both TCGA (Figure $2(\mathrm{~g})$, high $(n=82)$ vs. low $(n=247)$, $p<0.0001)$ and ICGC cohorts (Figure 2(h), high $(n=58)$ 
TABLE 1: Correlations between COPB2 mRNA expression and clinicopathological characteristics (logistic regression).

\begin{tabular}{lccc}
\hline Clinical characteristics & Total $(N)$ & OR in COPB2 expression & $p$ value \\
\hline Age (continuous) & 370 & $0.986(0.971-1.001)$ & 0.067 \\
Sex (male vs. female) & 370 & $0.726(0.468-1.122)$ & 0.150 \\
Status (with tumor vs. tumor-free) & 343 & $1.154(0.732-1.821)$ & 0.538 \\
Vascular invasion (positive vs. negative) & 314 & $1.000(0.627-1.594)$ & 1.000 \\
AFP (>20 vs. $\leq 20)$ & 277 & $1.616(1.006-2.606)$ & $0.048^{*}$ \\
T stage (T3+T2 vs. T1) & 354 & $1.539(1.013-2.345)$ & $0.044^{*}$ \\
Stage (III vs. I) & 256 & $1.744(1.033-2.969)$ & $0.038^{*}$ \\
Grade (G4+G3 vs. G2+G1) & 365 & $1.746(1.136-2.695)$ & $0.011^{*}$ \\
\hline
\end{tabular}

OR: odds ratio. ${ }^{*} p<0.05$.

TABLE 2: Univariate and multivariate analyses of the correlation of COPB2 with OS among HCC patients from TCGA cohort.

\begin{tabular}{|c|c|c|}
\hline Clinical characteristics & $\mathrm{HR}$ & $p$ value \\
\hline \multicolumn{3}{|l|}{ Univariate analysis } \\
\hline Age (continuous) & $1.250(0.882-1.772)$ & 0.210 \\
\hline Sex (male vs. female) & $0.805(0.564-1.147)$ & 0.230 \\
\hline BMI (continuous) & $0.974(0.941-1.007)$ & 0.124 \\
\hline $\mathrm{T}$ stage $(\mathrm{T} 4 / \mathrm{T} 3 / \mathrm{T} 2 / \mathrm{T} 1)$ & $1.665(1.390-1.993)$ & $<0.0001^{* * *}$ \\
\hline Lymph nodes (positive vs. negative) & $1.948(0.477-7.952)$ & 0.353 \\
\hline Distant metastasis (positive vs. negative) & $3.820(1.201-12.146)$ & $0.023^{*}$ \\
\hline Stage $(\mathrm{IV} / \mathrm{III} / \mathrm{II} / \mathrm{I})$ & $1.652(1.349-2.024)$ & $<0.0001^{* * *}$ \\
\hline Grade (G4/G3/G2/G1) & $1.127(0.892-1.424)$ & 0.317 \\
\hline Tumor status (with tumor vs. tumor-free) & $1.604(1.116-2.306)$ & $0.011^{*}$ \\
\hline Family cancer history (yes vs. no) & $1.182(0.819-1.707)$ & 0.372 \\
\hline New tumor event (yes vs. no) & $1.335(0.932-1.913)$ & 0.116 \\
\hline COPB2 expression (continuous) & $1.068(1.037-1.099)$ & $<0.0001^{* * *}$ \\
\hline \multicolumn{3}{|l|}{ Multivariate analysis } \\
\hline T stage $(\mathrm{T} 4 / \mathrm{T} 3 / \mathrm{T} 2 / \mathrm{T} 1)$ & $2.074(0.816-5.270)$ & 0.125 \\
\hline Distant metastasis (positive vs. negative) & $1.642(0.282-9.544)$ & 0.581 \\
\hline Stage (IV/III/II/I) & $0.772(0.269-2.215)$ & 0.630 \\
\hline Tumor status (with tumor vs. tumor-free) & $1.002(0.487-2.064)$ & 0.995 \\
\hline COPB2 expression (continuous) & $2.011(1.111-3.641)$ & $0.021^{*}$ \\
\hline
\end{tabular}

HR: hazard ratio. ${ }^{*} p<0.05$ and ${ }^{* * * *} p<0.0001$.

vs. low $(n=172), p<0.05)$. The upper quartile value of COPB2 expression levels was used as the cutoff point [22].

Univariate and multivariate Cox proportional hazard regression analyses were performed on TCGA cohort. In the univariate Cox analysis, shorter overall survival (OS) was found in those with higher expression of COPB2 (hazard ratio $(\mathrm{HR})=1.068,95 \%$ confidence interval (CI): 1.037-1.099, $p<0.0001)$, higher $\mathrm{T}$ stage $(\mathrm{HR}=1.665,95 \% \mathrm{CI}: 1.390$ $-1.993, p<0.0001)$, worse pathological stage $(\mathrm{HR}=1.652$, 95\% CI: $1.349-2.024, p<0.0001)$, and "with tumor" status $(\mathrm{HR}=1.604$, 95\% CI: 1.116-2.306, $p<0.05)$ (Table 2). However, in the multivariate Cox analysis, worse OS was only significantly associated with high expression of COPB2 ( $\mathrm{HR}=2.011$, 95\% CI: $1.111-3.641, p<0.05$ ) (Table 2). This indicates that COPB2 was an independent prognostic factor for HCC.
In summary, the above results indicated that high COPB2 expression correlated with poor prognosis in HCC.

3.3. GSEA Identified COPB2-Related Biological Signaling Pathways in HCC. To explore the biological signaling pathways involved in COPB2 expression in HCC, we performed GSEA of the high and low COPB2 expression groups in TCGA cohort. The results revealed a great number of significant differences (false discovery rate $(\mathrm{FDR})<0.05, p<0.05$ ) in the enrichment of the Molecular Signatures Database (MSigDB) Collection (c2.cp.kegg.v7.2.symbols.gmt), and we observed that the cell cycle (Figure 3(a), normalized enrichment score (NES): 2.114, FDR: 0.001, $p<0.001$ ), ERBB signaling pathway (Figure 3(b), NES: 2.065, FDR: 0.002, $p<0.001$ ), VEGF signaling pathway (Figure 3(c), NES: 


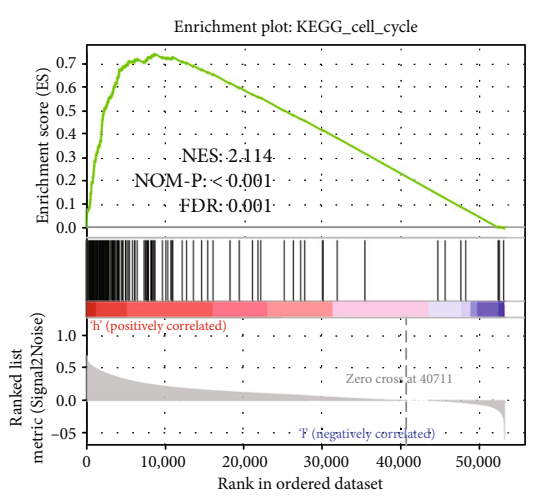

(a)

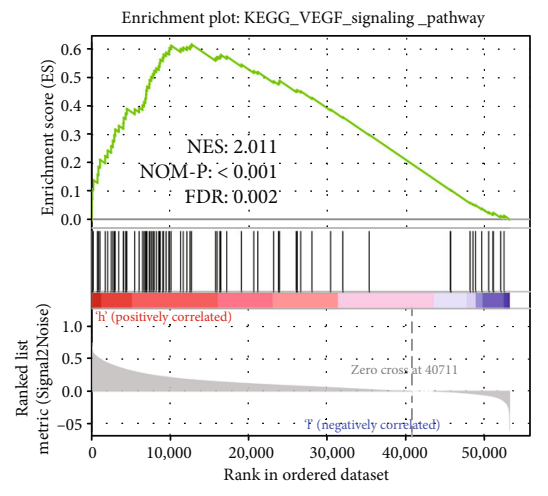

(c)

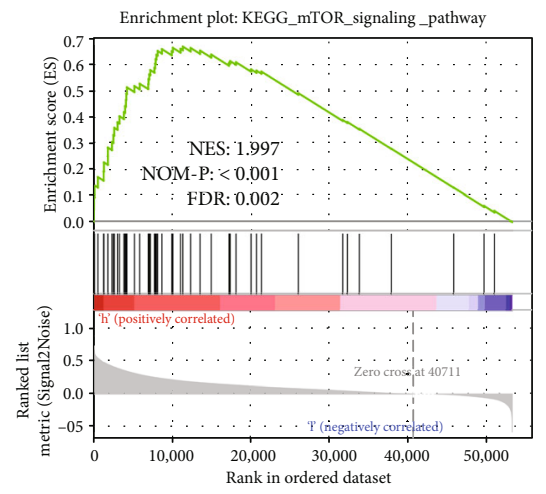

(e)

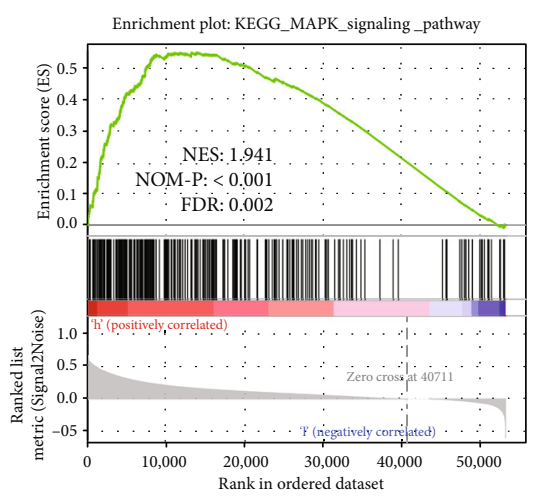

(g)

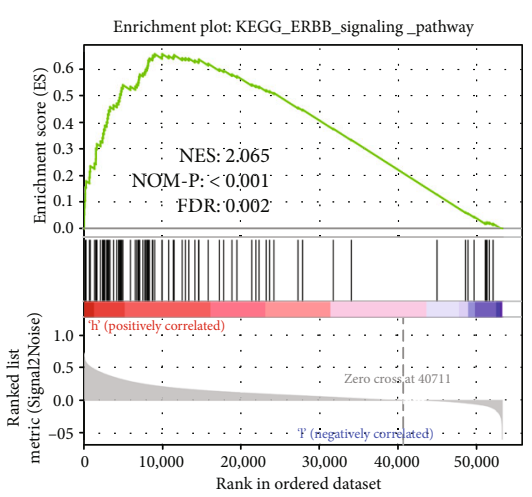

(b)

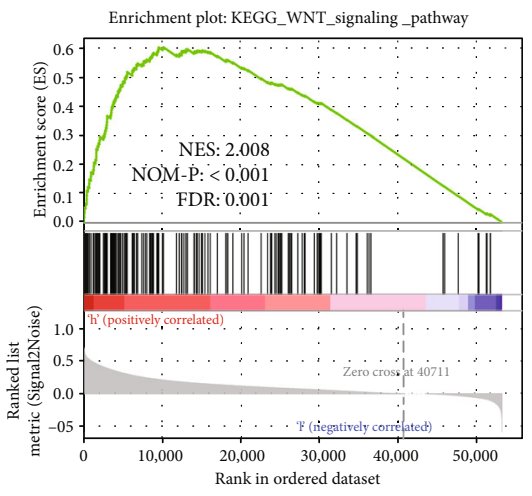

(d)

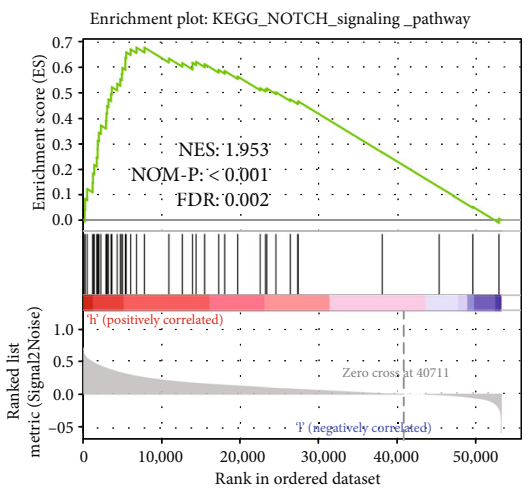

(f)

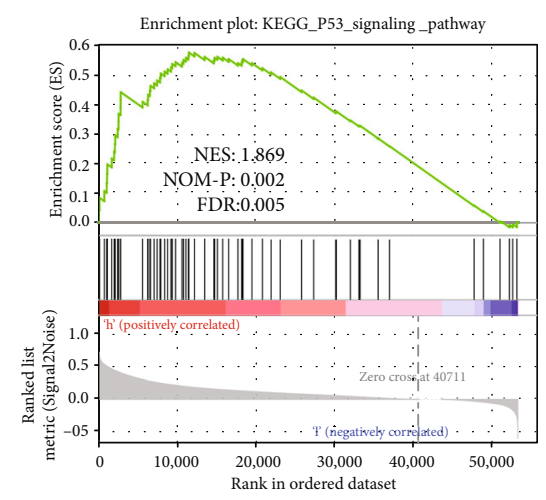

(h)

Figure 3: Continued. 


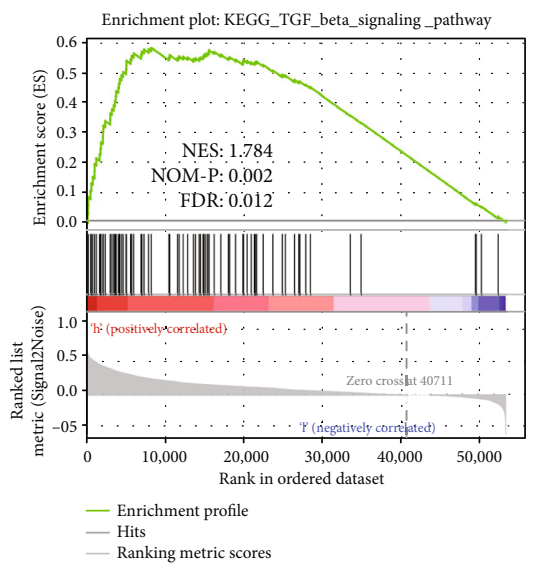

(i)

FIGURE 3: GSEA identified COPB2-related biological signaling pathways in HCC. (a) Cell cycle. (b) ERBB signaling pathway. (c) VRGF signaling pathway. (d) WNT signaling pathway. (e) mTOR signaling pathway. (f) NOTCH signaling pathway. (g) MAPK signaling pathway. (h) P53 signaling pathway. (i) TGF- $\beta$ signaling pathway. ES: enrichment score; NES: normalized ES; NOM-P: normalized $p$ value; FDR: false discovery rate.

2.011, FDR: $0.002, p<0.001)$, WNT signaling pathway (Figure 3(d), NES: 2.008, FDR: 0.001, $p<0.001$ ), mTOR signaling pathway (Figure 3(e), NES: 1.997, FDR: 0.002, $p<0.001$ ), NOTCH signaling pathway (Figure 3(f), NES: 1.953, FDR: $0.002, p<0.001)$, MAPK signaling pathway (Figure 3(g), NES: 1.941, FDR: 0.002, $p<0.001$ ), P53 signaling pathway (Figure 3(h), NES: 1.869, FDR: 0.005, $p<0.01$ ), and TGF- $\beta$ signaling pathway (Figure 3(i), NES: 1.784, FDR: $0.012, p<0.01)$ were differentially enriched in those with the high COPB2 mRNA expression phenotype. The results indicated that COPB2 may play a vital role in the occurrence and progression of HCC.

3.4. Knockdown of COPB2 Suppressed Migration and Invasion of HCC Cell Lines. GSEA results showed that overexpression of COPB2 positively correlated with the activation of many tumor-related pathways in HCC. To verify the results of GSEA, we performed a series of experiments at the cellular level. All experiments were repeated at least three times. Wound healing assays showed that the migration ability of the COPB2 knockdown group was significantly weaker than that of the vector-transfected control group in both the BEL7402 (Figures 4(a) and 4(b), $p<0.01$ ) and SMMC7721 (Figures $4(\mathrm{c})$ and $4(\mathrm{~d}), p<0.01$ ) cell lines. Transwell assays verified that downregulation of COPB2 significantly inhibited the migration (BEL7402, $p<0.001$; SMMC7721, $p<0.001$ ) and invasion (BEL7402, $p<0.001$; SMMC7721, $p<0.001$ ) abilities of both cell lines (Figures 4(e)-4(h)). Moreover, we also measured the change in epithelial-mesenchymal transition- (EMT-) related protein expression levels using western blotting assays. The results revealed that the protein level of E-cadherin was markedly elevated (BEL7402, $p<0.01$; SMMC7721, $p<0.01$ ), while the expression of N-cadherin (BEL7402, $p<0.001 ;$ SMMC7721, $p<0.001$ ), vimentin (BEL7402, $p<0.001 ;$ SMMC7721, $p<0.001$ ), and Snail (BEL7402, $p<0.001$; SMMC7721, $p<0.001$ ) was significantly downregulated in both cell lines after COPB2 knockdown (Figures 4(i)-4(k)).

3.5. Silencing of COPB2 Inhibits the Proliferation by Inhibiting mTOR Signaling. In order to explore whether COPB2 can affect the proliferation of HCC, we performed CCK-8 assays. As expected, cells transfected with siCOPB2 had a lower rate of proliferation than siNC-treated cells in both BEL7402 (Figure 5(a); $24 \mathrm{~h}: p<0.01,48 \mathrm{~h}: p<0.0001$, $72 \mathrm{~h}: p<0.0001$ ) and SMMC7721 (Figure 5(b); $24 \mathrm{~h}$ : $p<0.01,48 \mathrm{~h}: p<0.001,72 \mathrm{~h}: p<0.0001)$ cell lines. In addition, we examined their cell cycle distribution and observed that compared with the siNC group, there was a significant increase in the number of cells in the G0/G1 phase and a decrease in the number of cells in the G2/M phase in the siCOPB2 group in both the BEL7402 (Figure 5(c), G0/G1: $p<0.001$; G2/M: $p<0.001$ ) and SMMC7721 (Figure 5(d), G0/G1: $p<0.001$; G2/M: $p<0.001)$ cell lines. GSEA results suggested that the activation of the mTOR signaling pathway was closely associated with overexpression of COPB2 in HCC. To further confirm this, we performed immunohistochemical staining on tumor tissues and matched nontumor tissues in HCC patients. As expected, the results show that phospho-mTOR was significantly overexpressed in tumor tissues (Figures 5(e) and 5(f), $p<0.0001$ ). In addition, the activity of this pathway of HCC cell lines was examined using a western blotting assay. In the present study, we observed that after knocking down COPB2, the expression level of mTOR (BEL7402, $p<0.01$; SMMC7721, $p<0.01)$ and p70 S6K (BEL7402, $p<0.001$; SMMC7721, $p<0.001$ ) as well as phospho-mTOR (BEL7402, $p<0.01$; SMMC7721, $p<0.01$ ), phospho-p70 S6K (BEL7402, $p<0.01$; SMMC7721, $p<0.01)$, and their downstream protein cyclin D1 (BEL7402, $p<0.001$; SMMC7721, $p<0.001$ ) decreased in both cell lines (Figures 5(g)-5(i)). These results suggest that silencing of COPB2 inhibits cell proliferation and that the mTOR signaling pathway plays an important role. 


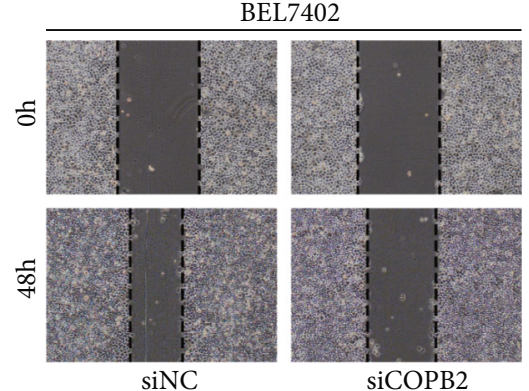

(a)

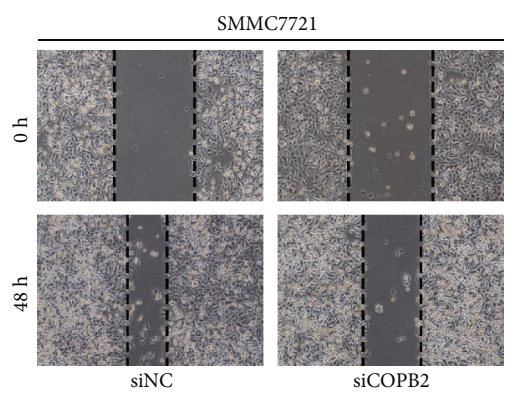

(c)

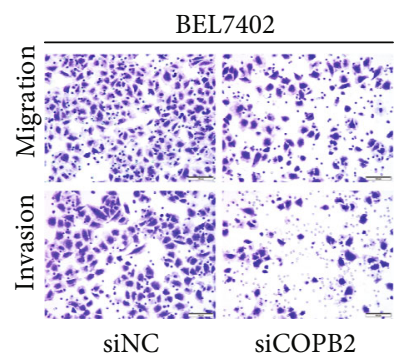

(e)

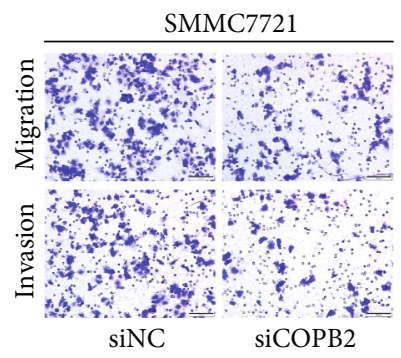

(g)

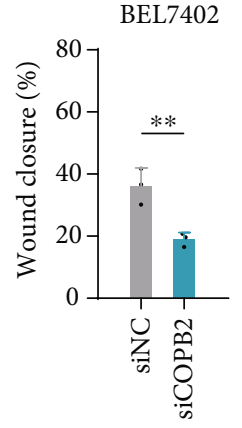

(b)

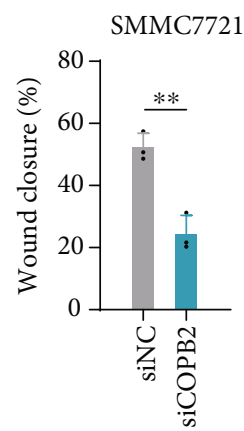

(d)

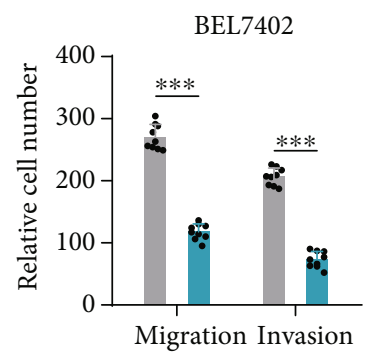

(f)

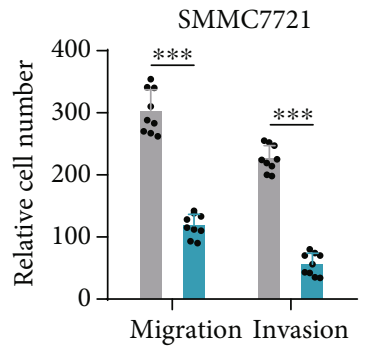

(h)

FIgure 4: Continued. 


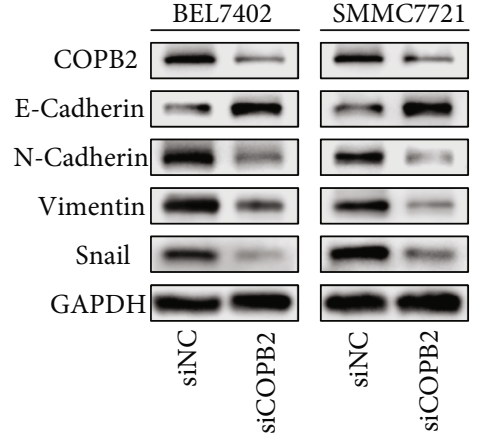

(i)

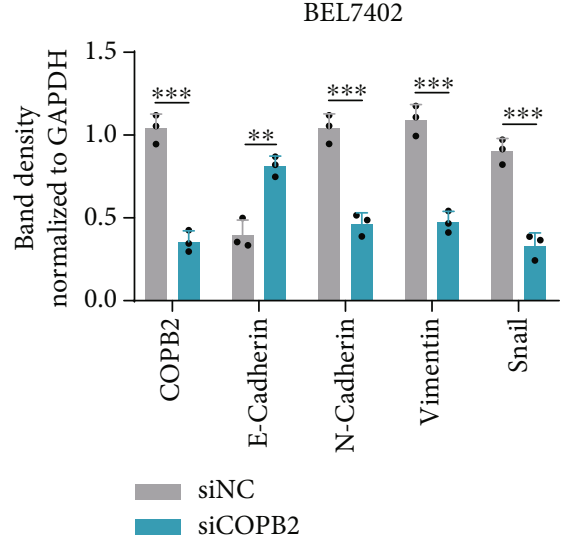

(j)

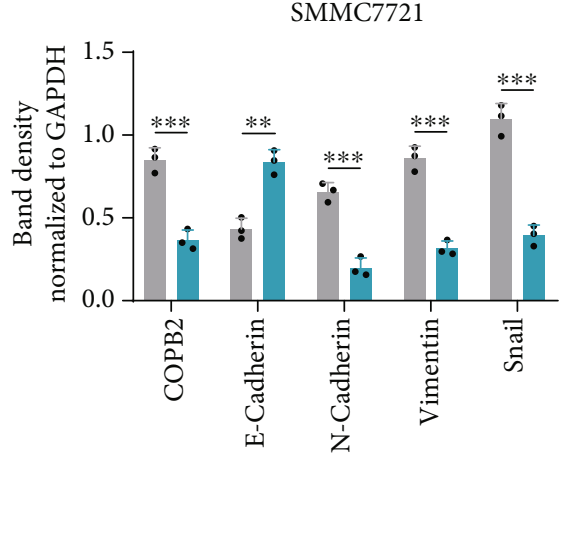

(k)

FIGURE 4: Knockdown of COPB2 suppressed migration and invasion in HCC cell lines. (a, c) Wound healing assays detected the cell migration ability of BEL7402 and SMMC7721 cells transfected with siNC or siCOPB2; the representative images were obtained at different time points. (b, d) Statistical analysis of the results of wound healing assays $(n=3)$. (e, g) Transwell assays were used to detect the cell migration and invasion ability of BEL7402 and SMMC7721 cells transfected with siNC and siCOPB2; the representative images are displayed. (f, h) Statistical analysis of the results of the Transwell assays $(n=9)$. (i) Representative images of western blotting analysis of COPB2, E-cadherin, N-cadherin, vimentin, Snail, and GAPDH in BEL7402 and SMMC7721 cells transfected with siNC and siCOPB2. GAPDH was used as the loading control. (j, k) Statistical analysis of gray values of western blotting assays $(n=3)$. All data were analyzed using the $t$-test and are displayed as mean \pm standard deviation (SD). ${ }^{*} p<0.05,{ }^{* *} p<0.01,{ }^{* * *} p<0.001$, and ${ }^{* * * *} p<0.0001$.

\section{Discussion}

HCC accounts for approximately $80 \%$ of primary liver cancers [1]. Due to its asymptomatic disease progression and lack of effective methods to make an early diagnosis, HCC is often diagnosed at an advanced stage [6]; its typically late-stage presentation, limited treatment options, and aggressive nature lead to it having a very poor prognosis [4, 24, 25]. In China, digestive tract cancers account for $36.4 \%$ of cancer-related deaths, of which liver cancer account for more than one-third [2]. Therefore, there is an urgent need to identify effective biomarkers for the diagnosis and prognosis of HCC, as well as therapeutic targets.

COPB2 is a $102 \mathrm{kDa}$ protein that was first identified in 1993 [26, 27]. Previous research confirmed that COPB2 is an element of non-clathrin-coated vesicles and is involved in regulating membrane transport in extracellular pathways $[9,28]$. In addition, as a subunit of the Golgi coatomer complex, COPB2 is essential for retrograde transport from the Golgi complex to the endoplasmic reticulum [11-13]. Compared with normal cells, the biosynthetic activity of tumor cells is abnormally vigorous [29]. As is well known, the Golgi complex plays an important role in anabolism; thus, COPB2 is certain to play a very important role in the occurrence and progression of tumors. Recently, the functions of COPB2 in tumors have been increasingly studied. In gliomas, COPB2 has been reported to be an important factor in the regulation of the immune microenvironment, and its high expression is related to adverse outcomes [14]. In breast cancer, COPB2 may predict metastasis [15]. In gastric cancer, COPB2 can affect the growth and apoptosis of gastric cancer cell lines via the RTK signaling pathway [30]. In lung adenocarcinoma, COPB2 was confirmed to be overexpressed and negatively correlated with survival, and COPB2 downregulation enhanced apoptosis and repressed proliferation and tumorigenesis in lung adenocarcinoma cells [31]. In prostate cancer, COPB2 has also been shown to be highly expressed and can promote PC-3 cell proliferation and inhibit apoptosis by affecting its cell cycle [16]. Downregulation of COPB2 could inhibit the growth of human cholangiocellular carcinoma cells [32]. It has also been reported that reduced editing of COPA, an important paralog of COPB2, has been implicated in the pathogenesis of HCC, and editing of COPA ${ }^{\mathrm{WT}}$ may switch it from a tumor-promoting gene to a tumor suppressor by deactivating the PI3K/AKT/mTOR pathway through downregulation of caveolin-1 (CAV1) [20]. These findings suggest an essential role of COPB2 in the occurrence and progression of tumors, which provides a good theoretical basis for our study of the role of COPB2 in HCC.

With the advancement of technology, high-throughput sequencing technology has been increasingly used in cancer research $[33,34]$. In the present study, we explored the role of COPB2 in human HCC and the underlying mechanism using database analysis combined with basic experiments. Bioinformatic analysis based on TCGA, ICGC, and GEO databases revealed that COPB2 mRNA levels were higher in HCC tissues than in nontumor tissues. At the same time, we confirmed the high expression of COPB2 protein in HCC tissues using immunohistochemical assay. The mRNA expression data and clinical information of HCC were then analyzed. Correlation and survival analyses showed that high COPB2 expression was closely correlated with advanced clinicopathological parameters (higher AFP, worse T stage, poor pathological stage, and higher grade) and worse prognosis. Univariate and multivariate Cox analyses indicated that COPB2 was an independent prognostic factor for HCC. GSEA suggested that various signaling pathways closely related to tumor occurrence and development [35-39] (e.g., 

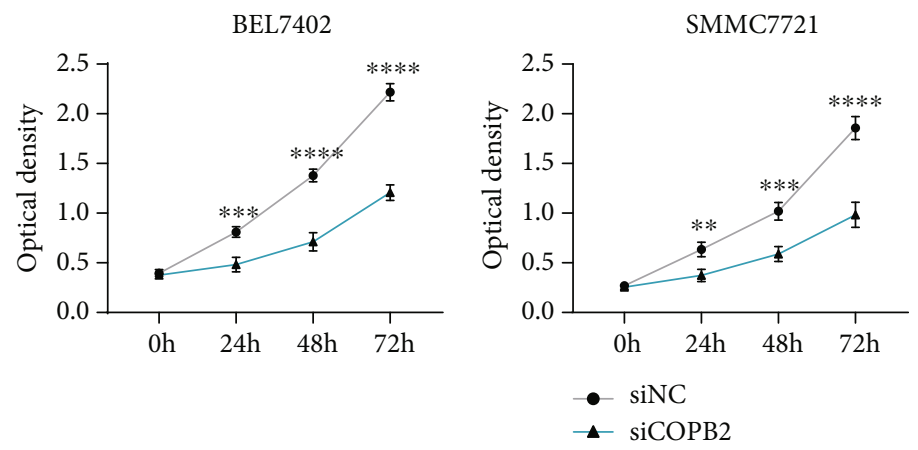

(a)

(b)
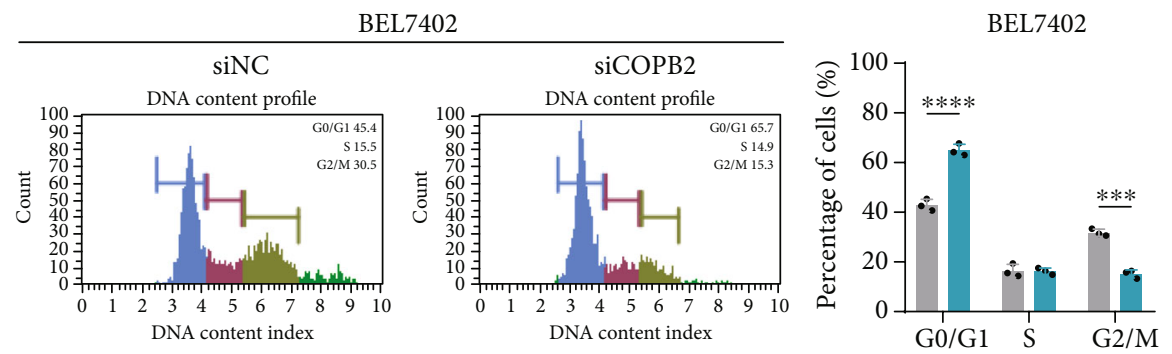

(c)
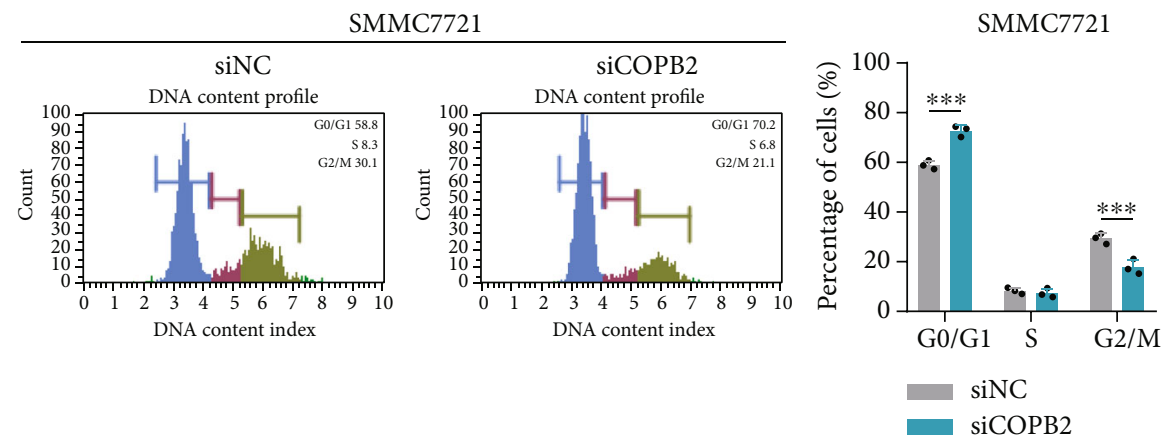

(d)

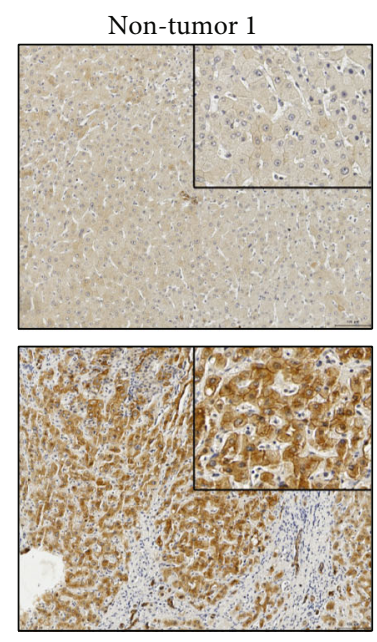

Tumor 1
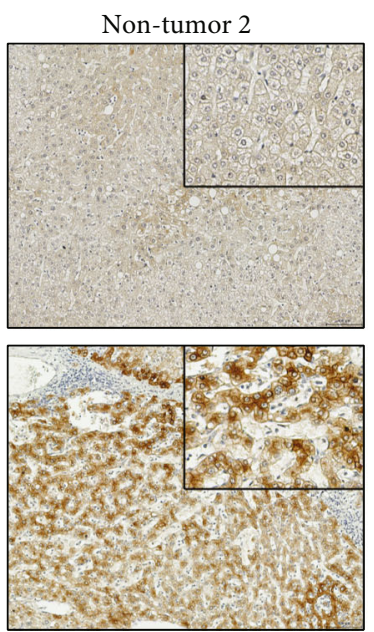

Tumor 2

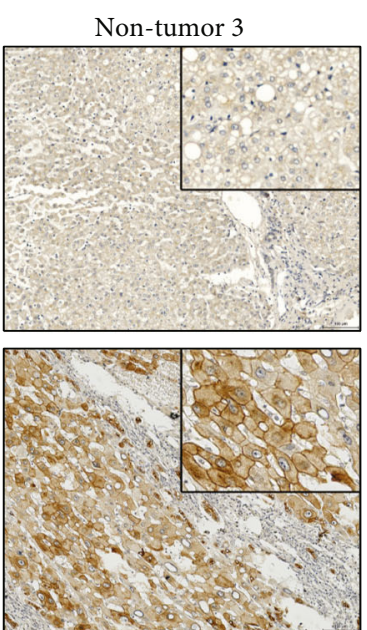

Tumor 3

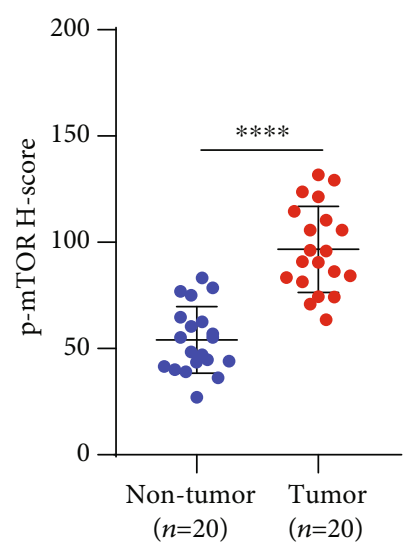

(e)

Figure 5: Continued. 


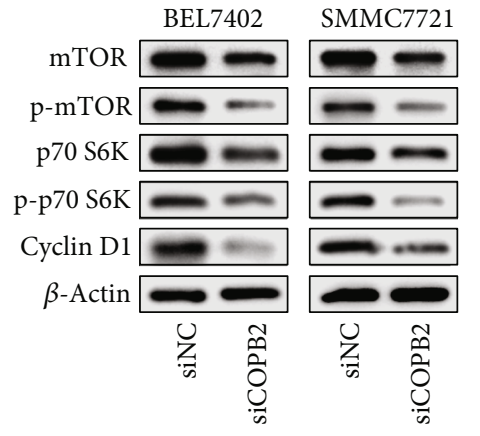

(g)

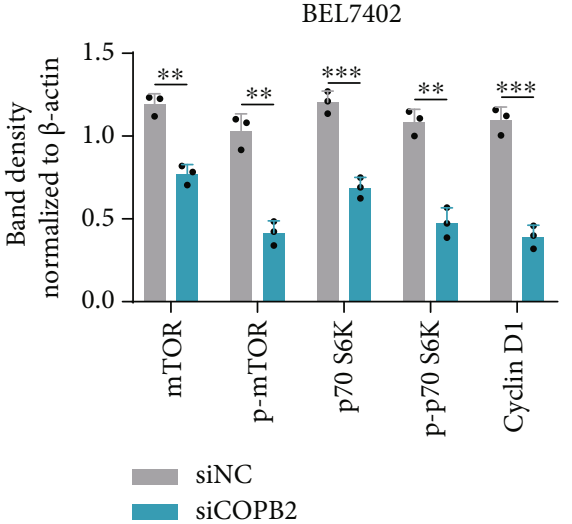

(h)

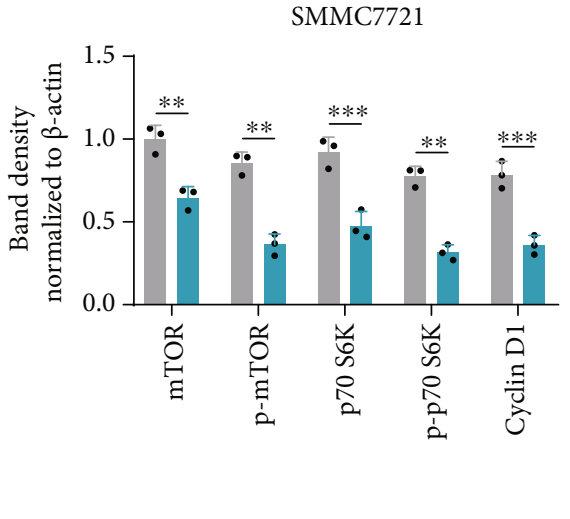

(i)

FIGURE 5: Silencing of COPB2 inhibits the proliferation by inhibiting mTOR signaling. (a, b) BEL7402 and SMMC7721 cells were transfected with siNC and siCOPB2, respectively, and cell viability was analyzed using the CCK-8 assays $(n=5)$ (two-way repeated measurement ANOVA with Sidak's multiple comparisons test). (c, d) Detection of the cell cycle distribution of BEL7402 and SMMC7721 cells after being transfected with siNC and siCOPB2 $(n=3)(t$-test). (e, f) Immunohistochemical analysis of p-mTOR in HCC tissues $(n=20)$ and adjacent nontumor tissues $(n=20)$ ( $t$-test). $(\mathrm{g})$ Representative images of western blotting analysis of mTOR, p-mTOR, p70 S6K, p-p70 S6K, cyclin D1, and $\beta$-actin in BEL7402 and SMMC7721 cells transfected with siNC and siCOPB2. \#x03B2;-actin was used as the loading control. (h, i) Statistical analysis of gray values of western blotting assays $(n=3)$ ( $t$-test). All data are displayed as mean \pm standard deviation (SD). ${ }^{*} p<0.05,{ }^{* *} p<0.01,{ }^{* * *} p<0.001$, and ${ }^{* * * *} p<0.0001$.

mTOR signaling pathway, WNT signaling pathway, VEGF signaling pathway, and $\mathrm{NOTCH}$ signaling pathway) were differentially enriched in those with the high COPB2 expression phenotype. To further explore the role of COPB2 in HCC, we performed a series of experiments. Functional investigations indicated that downregulation of COPB2 significantly inhibited the proliferation, migration, and invasion capacity of HCC in vitro. In addition, mechanistic experiments demonstrated that deletion of COPB2 significantly restrained EMT and activation of the mTOR signaling pathway.

The occurrence and progression of tumors are associated with abnormal regulation of multiple signaling pathways. EMT plays a vital role in tumorigenesis and tumor progression and is closely related to tumor invasion and migration abilities [40, 41]. Common signaling pathways, such as the WNT, NOTCH, MAPK, and TGF- $\beta$ signaling pathways, can activate EMT regulators [39, 42-44]. Evidence indicates that the mTOR signaling pathway governs cell growth and is activated in cancer $[35,45]$. The GSEA results showed that these signaling pathways were all enriched in the high COPB2 expression group in HCC, and the results of function and mechanism experiments are also consistent with this.

The results of the current study showed that COPB2 is overexpressed in HCC tissues, associated with HCC prognosis, and plays a crucial role in the proliferation, migration, and invasion of HCC cell lines in vitro, indicating that COPB2 is a novel prognostic biomarker and promising therapeutic target for HCC.

This study has some limitations. First, the tumor tissue specimens of the patients were usually obtained during surgery; however, patients with distant metastasis generally do not have indications for surgery. Consequently, the expression data from this patient population are rarely obtained. Second, according to the results of the GSEA, COPB2 may also influence the progression of HCC through other signal- ing pathways other than the mTOR signaling pathway; however, the current research on the relationship between COPB2 and HCC is in its infancy, and a lot of work is needed to explore whether COPB2 can affect HCC through other pathways in subsequent studies. Finally, this study only included cases from two cohorts, and a multicenter study should be conducted in the future.

\section{Conclusion}

COPB2 is a novel prognostic biomarker and a promising therapeutic target of HCC.
Abbreviations
HCC: Hepatocellular carcinoma
COPB2: Coatomer protein complex subunit beta 2
TCGA: The Cancer Genome Atlas
ICGC: International Cancer Genome Consortium
GEO: Gene Expression Omnibus
GSEA: Gene set enrichment analysis
AFP: Alpha fetoprotein
COPI: Coatomer complex I
COPs: Coat proteins
COPA: Coatomer protein complex subunit alpha
OS: Overall survival
ES: $\quad$ Enrichment score
NES: Normalized enrichment score
CCK-8: Cell counting kit-8
EMT: Epithelial-mesenchymal transition.

\section{Data Availability}

The data used to support this study are available from the corresponding author upon request. 


\section{Ethical Approval}

The present study was conducted according to the principles stated in the Declaration of Helsinki and approved by the Ethics Committee of Shandong Provincial Hospital, Cheeloo College of Medicine, Shandong University (No. 2019-032).

\section{Consent}

According to the guidelines of the ethics committee, written informed consent was obtained from all patients. All datasets were retrieved from public literature, and all written informed consent was obtained.

\section{Conflicts of Interest}

The authors report no conflicts of interest in this work.

\section{Authors' Contributions}

Jun Liu and Jiayao Zhang designed the research methods, performed the experiments, and analyzed the data. Xiaoyu Wang participated in experiments and data collection. Guangbing Li and Jingyi He collected tumor tissues. Ziwen $\mathrm{Lu}$, Yang Yang, Yong Jiang, Liyong Jiang, and Feiyu Li revised the manuscript. All authors have read and approved the final manuscript.

\section{Acknowledgments}

This study was supported by grants from the National Natural Science Foundation of China (No. 81373172 and No. 81770646).

\section{Supplementary Materials}

Table S1: the HCC patients' basic information of TCGA cohort. Table S2: the HCC patients' basic information of ICGC cohort. Table S3: basic characteristics of six HCC cohorts from GEO database. Figure S1: COPB2 expression analysis of four HCC cohorts from GEO database. (Supplementary Materials)

\section{References}

[1] F. Bray, J. Ferlay, I. Soerjomataram, R. L. Siegel, L. A. Torre, and A. Jemal, "Global cancer statistics 2018: GLOBOCAN estimates of incidence and mortality worldwide for 36 cancers in 185 countries," CA: a Cancer Journal for Clinicians, vol. 68, no. 6, pp. 394-424, 2018.

[2] R. M. Feng, Y. N. Zong, S. M. Cao, and R. H. Xu, "Current cancer situation in China: good or bad news from the 2018 Global Cancer Statistics?," Cancer Commun (Lond)., vol. 39, no. 1, p. 22, 2019.

[3] A. J. Craig, J. von Felden, T. Garcia-Lezana, S. Sarcognato, and A. Villanueva, "Tumour evolution in hepatocellular carcinoma," Nature Reviews. Gastroenterology \& Hepatology, vol. 17, no. 3, pp. 139-152, 2020.

[4] L. Kulik and H. B. El-Serag, "Epidemiology and management of hepatocellular carcinoma," Gastroenterology, vol. 156, no. 2, pp. 477-491.e1, 2019, e1.
[5] H. Guo, T. Wu, Q. Lu et al., "Surgical resection improves longterm survival of patients with hepatocellular carcinoma across different Barcelona Clinic Liver Cancer stages," Cancer Management and Research, vol. Volume 10, pp. 361-369, 2018.

[6] M. F. CHEDID, C. R. P. KRUEL, M. A. PINTO et al., "Hepatocellular carcinoma: diagnosis and operative management," Arquivos Brasileiros de Cirurgia Digestiva, vol. 30, no. 4, pp. 272-278, 2017.

[7] J. Hartke, M. Johnson, and M. Ghabril, "The diagnosis and treatment of hepatocellular carcinoma," Seminars in Diagnostic Pathology, vol. 34, no. 2, pp. 153-159, 2017.

[8] J. Best, "Novel implications in the treatment of hepatocellular carcinoma," Annals of Gastroenterology, vol. 30, no. 1, pp. 23-32, 2016.

[9] E. De Baere, F. Speleman, N. Van Roy, A. De Paepe, and L. Messiaen, "Assignment $<$ footref rid="foot 01 " $><$ sup $>1</$ sup $></$ footref $>$ of the cellular retinol-binding protein 1 gene (RBP1) and of the coatomer beta 'subunit gene (COPB2) to human chromosome band 3q23 by in situ hybridization," Cytogenetics and Cell Genetics, vol. 82, no. 3-4, pp. 226-227, 1998.

[10] R. Beck, M. Ravet, F. T. Wieland, and D. Cassel, "The COPI system: molecular mechanisms and function," FEBS Letters, vol. 583, no. 17, pp. 2701-2709, 2009.

[11] A. DiStasio, A. Driver, K. Sund et al., "Copb2 is essential for embryogenesis and hypomorphic mutations cause human microcephaly," Human Molecular Genetics, vol. 26, no. 24, pp. 4836-4848, 2017.

[12] M. L. Styers, A. K. O'Connor, R. Grabski, E. Cormet-Boyaka, and E. Sztul, "Depletion of beta-COP reveals a role for COPI in compartmentalization of secretory compartments and in biosynthetic transport of caveolin-1," American Journal of Physiology. Cell Physiology, vol. 294, no. 6, pp. C1485-C1498, 2008.

[13] F. Letourneur, E. C. Gaynor, S. Hennecke et al., "Coatomer is essential for retrieval of dilysine-tagged proteins to the endoplasmic reticulum," Cell, vol. 79, no. 7, pp. 1199-1207, 1994.

[14] Y. Zhou, X. Wang, X. Huang et al., "High expression of COPB2 predicts adverse outcomes: a potential therapeutic target for glioma," CNS Neuroscience \& Therapeutics, vol. 26, no. 3, pp. 309-318, 2020.

[15] A. Bhandari, C. Zheng, N. Sindan et al., "COPB2 is upregulated in breast cancer and plays a vital role in the metastasis via N-cadherin and vimentin," Journal of Cellular and Molecular Medicine, vol. 23, no. 8, pp. 5235-5245, 2019.

[16] Y. Mi, M. Yu, L. Zhang et al., "COPB2 is upregulated in prostate cancer and regulates PC-3 cell proliferation, cell cycle, and apoptosis," Archives of Medical Research, vol. 47, no. 6, pp. 411-418, 2016.

[17] Y. Wang, Z. Chai, M. Wang, Y. Jin, A. Yang, and M. Li, "COPB2 suppresses cell proliferation and induces cell cycle arrest in human colon cancer by regulating cell cycle-related proteins," Experimental and Therapeutic Medicine, vol. 15, no. 1, pp. 777-784, 2018.

[18] L. P. Jackson, M. Lewis, H. M. Kent et al., "Molecular basis for recognition of dilysine trafficking motifs by COPI," Developmental Cell, vol. 23, no. 6, pp. 1255-1262, 2012.

[19] N. Wong, A. Chan, S.-W. Lee et al., "Positional mapping for amplified DNA sequences on 1q21-q22 in hepatocellular carcinoma indicates candidate genes over-expression," Journal of Hepatology, vol. 38, no. 3, pp. 298-306, 2003. 
[20] Y. Song, O. An, X. Ren et al., "RNA editing mediates the functional switch of COPA in a novel mechanism of hepatocarcinogenesis," Journal of Hepatology, vol. 74, no. 1, pp. 135-147, 2021.

[21] X. Lu, P. Paliogiannis, D. F. Calvisi, and X. Chen, "Role of the mammalian target of rapamycin pathway in liver cancer: from molecular genetics to targeted therapies," Hepatology, vol. 73, no. S1, pp. 49-61, 2021.

[22] D. S. Chandrashekar, B. Bashel, S. A. H. Balasubramanya et al., "UALCAN: a portal for facilitating tumor subgroup gene expression and survival analyses," Neoplasia, vol. 19, no. 8, pp. 649-658, 2017.

[23] A. Subramanian, P. Tamayo, V. K. Mootha et al., "Gene set enrichment analysis: a knowledge-based approach for interpreting genome-wide expression profiles," Proceedings of the National Academy of Sciences of the United States of America, vol. 102, no. 43, pp. 15545-15550, 2005.

[24] R. L. Siegel, K. D. Miller, and A. Jemal, "Cancer statistics, 2019,” CA: a Cancer Journal for Clinicians, vol. 69, no. 1, pp. 7-34, 2018.

[25] M. C. Wallace, D. Preen, G. P. Jeffrey, and L. A. Adams, “The evolving epidemiology of hepatocellular carcinoma: a global perspective," Expert Review of Gastroenterology \& Hepatology, vol. 9, no. 6, pp. 765-779, 2015.

[26] K. J. Harrison-Lavoie, V. A. Lewis, G. M. Hynes, K. S. Collison, E. Nutland, and K. R. Willison, "A $102 \mathrm{kDa}$ subunit of a Golgiassociated particle has homology to beta subunits of trimeric $\mathrm{G}$ proteins," The EMBO Journal, vol. 12, no. 7, pp. 2847-2853, 1993.

[27] G. Stenbeck, C. Harter, A. Brecht et al., "beta' -COP, a novel subunit of coatomer," The EMBO Journal, vol. 12, no. 7, pp. 2841-2845, 1993.

[28] M. G. Waters, T. Serafini, and J. E. Rothman, “'Coatomer': a cytosolic protein complex containing subunits of nonclathrin-coated Golgi transport vesicles," Nature, vol. 349, no. 6306, pp. 248-251, 1991.

[29] R. J. DeBerardinis and N. S. Chandel, "Fundamentals of cancer metabolism," Science Advances, vol. 2, no. 5, p. e1600200, 2016.

[30] C. An, H. Li, X. Zhang et al., "Silencing of COPB2 inhibits the proliferation of gastric cancer cells and induces apoptosis via suppression of the RTK signaling pathway," International Journal of Oncology, vol. 54, no. 4, pp. 1195-1208, 2019.

[31] X. Pu, J. Wang, W. Li et al., “COPB2 promotes cell proliferation and tumorigenesis through up-regulating YAP1 expression in lung adenocarcinoma cells," Biomedicine \& Pharmacotherapy, vol. 103, pp. 373-380, 2018.

[32] Z. S. Li, C. H. Liu, Z. Liu, C. L. Zhu, and Q. Huang, "Downregulation of COPB2 by RNAi inhibits growth of human cholangiocellular carcinoma cells," European Review for Medical and Pharmacological Sciences, vol. 22, no. 4, pp. 985-992, 2018.

[33] M. A. Doyle, J. Li, K. Doig, A. Fellowes, and S. Q. Wong, "Studying cancer genomics through next-generation DNA sequencing and bioinformatics," Methods in Molecular Biology, vol. 1168, pp. 83-98, 2014.

[34] M. Meyerson, S. Gabriel, and G. Getz, "Advances in understanding cancer genomes through second-generation sequencing," Nature Reviews. Genetics, vol. 11, no. 10, pp. 685-696, 2010 .
[35] A. K. Murugan, "mTOR: role in cancer, metastasis and drug resistance," Seminars in Cancer Biology, vol. 59, pp. 92-111, 2019.

[36] O. Meurette and P. Mehlen, "Notch signaling in the tumor microenvironment," Cancer Cell, vol. 34, no. 4, pp. 536-548, 2018.

[37] T. Zhan, N. Rindtorff, and M. Boutros, "Wnt signaling in cancer," Oncogene, vol. 36, no. 11, pp. 1461-1473, 2017.

[38] L. K. Boroughs and R. J. DeBerardinis, "Metabolic pathways promoting cancer cell survival and growth," Nature Cell Biology, vol. 17, no. 4, pp. 351-359, 2015.

[39] S. Lamouille, J. Xu, and R. Derynck, "Molecular mechanisms of epithelial-mesenchymal transition," Nature Reviews. Molecular Cell Biology, vol. 15, no. 3, pp. 178-196, 2014.

[40] Y. Zhang and R. A. Weinberg, "Epithelial-to-mesenchymal transition in cancer: complexity and opportunities," Frontiers in Medicine, vol. 12, no. 4, pp. 361-373, 2018.

[41] M. A. Nieto, R. Y.-J. Huang, R. A. Jackson, and J. P. Thiery, "Emt: 2016," Cell, vol. 166, no. 1, pp. 21-45, 2016.

[42] A. Dongre and R. A. Weinberg, "New insights into the mechanisms of epithelial-mesenchymal transition and implications for cancer," Nature Reviews. Molecular Cell Biology, vol. 20, no. 2, pp. 69-84, 2019.

[43] V. Mittal, "Epithelial mesenchymal transition in tumor metastasis," Annual Review of Pathology, vol. 13, no. 1, pp. 395-412, 2018.

[44] P. Savagner, "Leaving the neighborhood: molecular mechanisms involved during epithelial-mesenchymal transition," BioEssays, vol. 23, no. 10, pp. 912-923, 2001.

[45] D. Mossmann, S. Park, and M. N. Hall, "mTOR signalling and cellular metabolism are mutual determinants in cancer," Nature Reviews. Cancer, vol. 18, no. 12, pp. 744-757, 2018. 